\title{
Cranial Nerve Palsy Secondary to Botulism After Black Tar Heroin Use
}

\author{
Hiroshi T. Suzuki, MD, MPH and Hari Reddy, MD
}

Introduction: Wound botulism (WB) is an uncommon but severe neuromuscular illness caused by the bacterium Clostridium botulinum in an infected wound. There has been a dramatic increase in WB associated with black tar heroin injection in California.

Case Description: A 50-year-old male with heroin abuse presented to the emergency department with a 2day history of dysphagia and dysarthria. Physical examination revealed slurred speech, inability to manipulate tongue, and slowed eye movements. The patient was also noted to have progressive weakness during hospitalization. Laboratory findings were unremarkable, and further workup, including a computerized tomography scan of the head and soft neck tissue, showed no abnormal findings. Given the history of heroin abuse in Southern California and findings on physical examination, a diagnosis of WB needed to be considered as the differential. The Department of Health was contacted, and treatment was initiated with botulism antitoxin and metronidazole. Despite the treatment, the patient's condition did not improve, and the patient died. The resulting diagnosis was confirmed by $C$. botulism toxin A found in his serum a few days after the patient died.

Discussion: Progressive cranial nerve palsy with symmetric descending paralysis with heroin abuse should raise the suspicion of WB and require prompt diagnosis and treatment. This case highlights raising awareness of the disease could help lead to early diagnosis and treatment. ( $\mathrm{J}$ Am Board Fam Med 2020;34:808-810.)

Keywords: Botulism, Case Report, Community Medicine, Critical Care, Family Medicine, Heroin Addiction, Infectious Diseases, Narcotics, Neurology, Opiates, Population Health, Primary Health Care

\section{Case Report}

A 50-year-old male with a past medical history of polysubstance dependence with heroin and methamphetamine presented to the emergency department in San Bernardino County, Southern California, with a 2day history of dysphagia and dysarthria. At the time, the patient could follow commands, and there was no history of fever, paresthesia, or respiratory difficulty.

On examination, vital signs were within normal limits. The patient appeared lethargic and had slurred speech. He had slowed eye movements, but pupils were reactive with no evidence of ptosis.

This article was externally peer reviewed.

Submitted 24 December 2020; revised 16 March 2021; accepted 23 March 2021.

From the University of California, Riverside, Department of Family Medicine, University of California, Riverside (HS); St. Bernardine Medical Center, Sound Critical Care, San Bernardino, CA (HR)

Funding: None.

Conflict of interest: None.

Corresponding author: Hiroshi T. Suzuki MD, MPH, University of California, Riverside, School of Medicine Family Medicine, 1890 N Waterman Ave, San Bernardino, CA 92404, Phone: 909- 881-5145 (E-mail: hiroshi.suzuki@medsch.ucr.edu).
Facial muscle power was normal, and the patient had a normal gag reflex. Cranial nerves I through $\mathrm{XI}$ were intact, but he was unable to manipulate his tongue. Proximal and distal muscle strength was intact in the upper and lower limbs. Deep tendon reflexes were preserved, and sensation was intact.

A complete blood cell count was within normal limits. The results of the basic metabolic panel and liver function tests were within normal limits. Blood cultures were negative. Urine toxicology was positive for opiates and amphetamine. Electrocardiogram revealed normal sinus rhythm with a rate of 89 beats per minute. A computerized tomography (CT) scan of the head did not reveal evidence of intracranial bleed or mass. A CT scan of his soft neck tissue was done to assess for retropharyngeal abscess, which was negative for acute pathology.

The patient was initially admitted to the telemetry unit with further workup for possible ischemic stroke, including carotid doppler, echocardiogram, and magnetic resonance imaging (MRI) of the brain. After a few hours, he went into pulseless electric activity arrest, received 3 rounds of cardiopulmonary 
resuscitation with the return of spontaneous circulation in 11 minutes. He was subsequently intubated and admitted to an intensive care unit (ICU) for cardiac arrest and inability to protect the airway. He was cooled for 24 hours with the targeted temperature management to $33 \mathrm{C}^{\circ}$. After 24 hours, he was rewarmed for neurological assessment; however, an episode of seizure on electroencephalogram was consistent with status epilepticus. He was managed with multiple antiepileptic drugs, propofol and versed drips, and the ultimate need for burst suppression with phenobarbital. After cessation of seizure activity, he had sluggishly reactive pupils, no corneal reflexes present, and no gag reflex appreciated. Given his examination findings and prolonged status epilepticus, it was thought that he had hypoxic-ischemic encephalopathy (HIE). However, given his bulbar symptoms on presentation and weakness, $\mathrm{WB}$ was entertained, especially given his heroin use. Accordingly, the San Bernardino County Public Health Department was contacted and sent over the botulism antitoxin, which was promptly given. The culture was sent over to the Department of Public Health. He was also started on metronidazole. On day 7 , he did not have a meaningful neurologic response after he was off sedation for over 48 hours. MRI was obtained concerning for HIE, showing changes consistent with HIE. Given his poor prognosis, palliative care discussions were had with his mother, and comfort care was initiated. He died on the same day. A few days after he died, the health department reported C. botulism toxin A in his serum.

\section{Discussion}

WB is a rare but serious paralytic illness caused by a toxin produced by the bacterium C. botulinum in an infected wound. Early cases were seen with open wounds caused by crush injuries, gunshot wounds, and open fractures. The first reported WB case due to subcutaneous drug use was in 1982 in New York City. ${ }^{1}$ In recent years, California has experienced an epidemic of this form of botulism, with nearly $82 \%$ of all WB cases in the country between 2001 and 2016 from this region (as reported to the Centers for Disease Control and Prevention). ${ }^{2}$ This increase in botulism incidence has coincided with an epidemic of black tar heroin (BTH) use in California. From 2001 to 2005, there was an average of 3 cases of WB per year in Los Angeles (LA) County. From 2009 to 2012, there were 50 confirmed WB cases in California; 49 cases were associated with intravenous heroin use. ${ }^{3}$ Between 2013 and 2017, 14 confirmed cases of WB have occurred in LA County alone. Eight cases were associated with BTH, 4 with heroin, and 2 with cocaine and methamphetamine. ${ }^{4}$ More recently, there was a WB outbreak in San Diego County between September 2017 and April 2018, where 9 cases have been reported. Seven of 9 cases were associated with BTH, and 2 were associated with heroin use. ${ }^{2}$

BTH is a dark, gummy drug mainly produced in Mexico and often contains unusual diluents to increase bulk or contaminants, including shoe polish and dirt. ${ }^{2}$ However, it is still very rare for heroin users to have WB. The populations of patients most prone are intramuscular or subcutaneous heroin users. Administration by this method is conducive to infection, abscess formation, and the anaerobic medium needed for germination of botulism spores.

There are 7 recognized types of botulism neurotoxins (types A thorough G), of which only A, B, E, F cause human disease. Toxin $\mathrm{A}$ is responsible for about $80 \%$ of all $\mathrm{WB}$ cases, whereas the remaining $20 \%$ are caused predominantly by Toxin B. ${ }^{5}$ The Toxin acts presynaptically at all peripheral cholinergic synapses blocking neuromuscular junctions as well as parasympathetic and sympathetic pathways. ${ }^{6}$ Cranial nerves are usually affected before descending symmetrical skeletal muscle weakness. ${ }^{6}$ Typically, early symptoms include blurry vision, diplopia, dysphagia, and dysarthria followed by limb paralysis and respiratory compromise. ${ }^{6}$ Diaphragmatic and accessory muscle weakness leading to respiratory failure usually requires prolonged respiratory support. Botulism toxin is considered the most potent lethal substance known. It is 15,000 to 100,000 times more toxic than Sarin, the potent organophosphate nerve agent used in a terrorist attack on Tokyo subway system. ${ }^{7}$

Botulism is often underdiagnosed as many clinicians are not familiar with the disease process, and many symptoms can be mistaken for more common clinical entities. If there is even slight suspicion of botulism, the local health department should be contacted. Serum should then be sent for mouse lethality assays and wound exudate or tissue sample should be obtained for anaerobic culture. Toxin type is determined by neutralizing the activity of toxic samples injected into mice with type-specific botulism AT. ${ }^{7}$ The differential diagnosis includes Miller Fischer variant of Guillain-Barre syndrome 
(GBS), myasthenia gravis (MG), Eaton-Lambert syndrome, stroke syndrome, tick paralysis, opioid overdose, organophosphosphate intoxication, shellfish poisoning, diphtheria, and carbon monoxide toxicity. Diagnosis is supported by ancillary testing such as normal MRI results to rule out stroke, LP to rule out GBS, and tensilon test to rule out MG (though botulism can produce weak positive results). If negative and there is strong clinical suspicion for $\mathrm{WB}$, the local health department should be contacted for urgent antitoxin administration. EMG helps reveal decreased amplitude of action potentials in affected muscle groups and incremental increase in amplitude to rapid repetitive electromyography.

With the advent of ventilators and the modern ICU, WB has a $10 \%$ to $15 \%$ mortality rate compared with $60 \%$ to $70 \%$ in the early $20^{\text {th }}$ century. ${ }^{6}$ As mentioned earlier, botulism AT should be given as soon as there is any clinical suspicion. The AT binds to free, not intraneuronal, neurotoxin and prevents worsening of the paralysis. ${ }^{6}$ A single trivalent (against A, B, and E) or heptavalent (against all types) antitoxin neutralizes all free toxins and has a half-life of 5 to 8 days. $^{6}$ The toxin may cause a hypersensitivity reaction, and skin testing before administration is suggested. A retrospective analysis of 134 cases of type A botulism showed an overall mortality rate of $10 \%$ with patients who received early treatment ( $<4$ hours), $15 \%$ with those who received late treatment ( $>24$ hours), and $46 \%$ with those who received no treatment. ${ }^{7}$ A study looking at a recent foodborne botulism outbreak in Thailand revealed that early AT administration significantly reduced days needed on the ventilator. ${ }^{8}$ Another measure that has shown decreased mortality rates over the past 40 years is supportive care with mechanical ventilation. If a patient is suspected of having $\mathrm{WB}$, they should be monitored in an ICU from the outset. In addition, vital capacity should be checked daily at the earliest sign of respiratory compromise. Lastly, the wound must undergo surgical debridement followed by antibiotic administration of penicillin $G$ or metronidazole if allergic to penicillin.

In our case, only cranial nerve symptoms were observed on arrival. The finding in the setting of injection drug use raised the suspicion for $\mathrm{WB}$, and the decision of administrating AT was made. Although $\mathrm{WB}$ is still a very rare disease even in chronic heroin users, public health should be contacted when the patient with chronic BTH use presented with cranial nerve palsy. Injection drug users should be made aware of the new dangers of heroin use and taught methods that promote harm reduction. Ultimately, prompt recognition by physicians in the community is needed to prevent increased mortality, hospital stay, and rehabilitation time.

To see this article online, please go to: http://jabfm.org/content/ 34/4/808.full.

\section{References}

1. Merrison AFA, Chidley KE, Dunnett J, Sieradzan KA. Wound botulism associated with subcutaneous drug use. BMJ 2002;325:1020-1021.

2. Peak CM, Rosen H, Kamali A, et al. Wound botulism outbreak among persons who use black tar heroin - San Diego County, California, 2017-2018. MMWR 2019/;67:1415-1418.

3. The California Department of Public Health. Epidemiologic Summary of Wound Botulism in California, 2009-2012: Available from: https:// www.cdph.ca.gov/Programs/CID/DCDC/CDPH \%20Document\%20Library/WoundBotulismEpi Summary09-12.pdf. Published November 14, 2014. Accessed February 15, 2021.

4. County of Los Angeles Public Health: Los Angeles County Department of Public Health Health Alert: Wound Botulism Cases Associated with Heroin. Los Angeles County Health Alert Network 2018. Available from: http://publichealth.lacounty.gov/eprp/Health\% 20Alerts/DPH\%20HAN\%20Alert\%20Wound\% 20Botulism \%200702 18\%20FINAL.pdf. Published July 2, 2018. Accessed February 15, 2021.

5. Pujar T, Spinello IM. A 38-year-old woman with heroin addiction, ptosis, respiratory failure, and proximal myopathy. Chest 2008;134:867-870.

6. Vera J, Hensiek A, Woodrow C, et al. Opthalmoplegia and slurred speech in an intravenous drug user. PLoS Med 2006;3:e453-e2211.

7. Shapiro RL, Hatheway C, Sherdlow DL. Botulism in the United States: a clinical and epidemiologic review. Ann Intern Med 1998;129:221-228.

8. Kongsaengdao S, Samintarapanya K, Rusmeechan S, et al. Thai Botulism Study Group. An outbreak of botulism in thailand: clinical manifestations and management of severe respiratory failure. CID 2006; 43:1247-1256. 